\title{
XXII. On the method of the least squares
}

\author{
J. Ivory Esq. M.A. F.R.S.
}

To cite this article: J. Ivory Esq. M.A. F.R.S. (1826) XXII. On the method of the least squares, Philosophical Magazine Series 1, 68:341, 161-165, DOI: 10.1080/14786442608674103

To link to this article: http://dx.doi.org/10.1080/14786442608674103

曲 Published online: 10 Aug 2009.

Submit your article to this journal $\pi$

Џll Article views: 6

Q View related articles $\asymp$

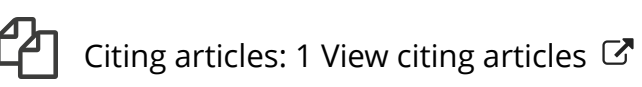




\title{
PHILOSOPHICAL MAGAZINE AND JOURNAL.
}

\author{
$30^{\text {th }} \quad S E P T E M B E R \quad 1826$.
}

XXII. On the Method of the Least Squares. By J. Ivory, Esq. M.A. F.R.S.*

I HAVE already treated of the method of the least squares 1 in this Journal for January 1825, and the two following months. By altering a little an idea first suggested by Cotes, we may represent the errors of observation by the parts of a rigid line, the positive errors being on one side, and the negative errors on the other side, of a common fulcrum; and if we append to the extremities of the errors, weights proportional to the coefficients of the correction in the equations of condition, the equilibrium of the weights is the rule of the least squares, and determines the most advantageous value of the correction sought. When there are several elements to be corrected simultaneously, the coefficients of the different corrections in the equations of condition will form so many separate sets of weights, and the levers must be in equilibrio whichever set is appended; by which means we obtain as many equations as there are unknown quantities to be found. This method is at least precise and free from every thing conjectural or tentative. It is likewise founded on just principles; for every coefficient in the equations of condition has its due influence in the quantity of the result. But it must be allowed that the introducing of the properties of the lever and of equilibrium in a demonstration of this kind is not altogether unexceptionable. Such considerations would not be necessary if the truth to be proved were entirely disengaged from what is foreign to it. It is however extremely desirable that a method, which is of great practical utility, should be clearly and simply deduced from the real principles alone concerned; because it is only when this is done that the method can be fully understood, and that we can apply it with confidence and without danger of mistake. My intention in returning to this subject is to attempt an explanation of the ground of the method of the least squares that may in some degree answer the description here given.

* Communicated by the Author.

Vol. 68. No. 341. Sept. 1826. 
In the most simple case of only one element the equations of condition are as follows; viz.

$$
\begin{aligned}
& e=a x-m \\
& e^{\prime}=a^{\prime} x-m^{\prime} \\
& e^{\prime \prime}=a^{\prime \prime} x-m^{\prime \prime}
\end{aligned}
$$

\&c.

in which the coefficients of the correction $x$, viz. $a, a^{\prime}, a^{\prime \prime}, \& \mathrm{c}$. are all positive. Now it is manifest that the errors $e, e^{\prime}, e^{\prime \prime}, \& \mathrm{c}$. depend upon the correction $x$; in so much that when any particular value is assigned to $x$, all the errors are immediately determined. It appears therefore that there can exist no reason for preferring one value of $x$ to another, except the nature of the errors, or the general character impressed upon them on the supposition that the experiments are skilfully executed. When the law of the errors is fulfilled, and when, besides their quantity is confined within the least possible limits, the problem is solved, and we have found that value of the correction which nust be preferred to every other. What then is the general character of the errors of a set of experiments made for the purpose of ascertaining the quantity of some physical magnitude, or of approximating to it more nearly than had been done before? We may suppose at least, that the experiments are liable only to irregular and fortuitous errors; that every cause tending to make the results of observation incline more to one side than to another, has been carefully investigated and removed; and in short, that the errors contain no constant part common to them all. What is here said must not be understood exclusively of the case when every error contains a part of the same magnitude affected with the same sign; the principle evidently extends to all cases when the errors contain parts affected with the same sign although unequal in magnitude, provided these parts are necessarily connected with one another so that they must exist simultaneously. The point does not turn upon the equality or inequality of the parts, but upon this, That the errors of separate experiments must be independent on another and subject to no determinate law. In a repetition of the same experiment it is universally the practice to take the arithmetical mean of all the observed quantities, as much more exact than any particular result. Now this rule is founded on the independence of the experiments and of the errors to which they are liable; whence it follows that the errors in excess may be expected to balance those in defect; since no reason can be assigned why the amount of one should be different from the amount of the other. When the quantity to be observed varies from one experiment to another, always containing, however, 
the same elementary quantities sought, the principle of the independence of the experiments will still hold good; we must still suppose that the error committed in one case has no influence whatever on the error committed in any other case; and I shall prove that this principle, combined with the equations of condition, leads necessarily to the method of the least squares, of which the rule for the arithmetical mean is only a particular case.

Multiply all the terms of every equation of condition by the coefficient of $x$; then, having added all the results, we shall get

$$
\mathbf{S}(a e)=x \times \mathbf{S}\left(a^{2}\right)-\mathbf{S}(a m)
$$

the symbols used for the sake of abridging being thus explained, viz. $\quad \mathrm{S}(a e)=a e+a^{\prime} e^{l}+a^{\prime \prime} e^{\prime \prime}+\& \mathrm{cc}$.

$$
\begin{aligned}
& \mathbf{S}\left(a^{2}\right)=a^{2}+a^{\prime 2}+a^{\prime \prime 2}+\& \mathrm{c} . \\
& \mathbf{S}(a m)=a m+a^{\prime} m^{\prime}+a^{\prime \prime} m^{\prime \prime}+\& \mathrm{c} .
\end{aligned}
$$

Find $x$ from the equation just obtained, then

$$
x=\frac{\mathrm{S}(a m)}{\mathrm{S}\left(a^{2}\right)}+\frac{\mathrm{S}(a e)}{\mathrm{S}\left(a^{2}\right)},
$$

and by substituting this value in the expressions of the errors, we further obtain,

$$
\begin{aligned}
& e=-m+a \cdot \frac{\mathrm{S}(a m)}{\mathrm{S}\left(a^{2}\right)}+a \cdot \frac{\mathrm{S}(a e)}{\mathrm{S}\left(a^{2}\right)}, \\
& e^{\prime}=-m^{\prime}+a^{\prime} \cdot \frac{\mathrm{S}(a m)}{\mathrm{S}\left(a^{2}\right)}+a^{\prime} \cdot \frac{\mathrm{S}(a e)}{\mathrm{S}\left(a^{2}\right)}, \\
& \text { \&c. }
\end{aligned}
$$

All the errors as well as $-x$ now depend upon one and the same quantity, namely, $\mathrm{S}(a e)$. If we make this arbitrary quantity go through every gradation of magnitude, we shall obtain ail the possible systems of the errors and every possible value of $x$. Let $\varepsilon, \varepsilon^{\prime}, \varepsilon^{\prime \prime} \& c$. denote the particular set of errors found by the condition, $\quad S(a \varepsilon)=0$;

which equation may be otherwise written thus,

$$
\varepsilon \frac{d s}{d x}+\varepsilon^{\prime} \frac{d \varepsilon^{\prime}}{d x}+\varepsilon^{\prime \prime} \frac{d \varepsilon^{\prime \prime}}{d x}+8 \mathrm{cc} .=0,
$$

and it determines the minimum of the expression $\varepsilon^{2}+\varepsilon^{2}+$ $\varepsilon^{\prime \prime}+\& c .:$ it also fulfils the equilibrium of the weights $a, a^{r}, a^{\prime \prime}$ \&c. as noticed in the beginning of this paper. Now put $\xi$ for the particular value of $x$ when the errors are $z, \varepsilon^{\prime}, \varepsilon^{\prime \prime} \& c$.; then, by making $S(a e)=0$ in the foregoing formulæ, we get

$$
\begin{aligned}
& \xi=\frac{\mathbf{S}(a m)}{\mathbf{S}\left(a^{2}\right)} \\
& \xi=-m+a \cdot \frac{\mathbf{S}(a m)}{\mathbf{S}\left(a^{2}\right)}, \\
& \xi^{\prime}=-m^{\prime}+a^{\prime} \cdot \frac{\mathbf{S}(a m)}{\mathbf{S}\left(a^{2}\right)}, \\
& \varepsilon^{\prime \prime}=-m^{\prime \prime}+a^{\prime \prime} \cdot \frac{\mathbf{S}(a m)}{\mathbf{S}\left(a^{2}\right)}, \\
& \& \mathrm{c} .
\end{aligned}
$$


These expressions show that $\varepsilon, \varepsilon^{\prime}, \varepsilon^{\prime \prime}$, \&c. are independent of one another. Every one is singly derived from the quantities of its proper experiment, without being influenced by the magnitudes of the rest. Let us now suppose that $S(a e)$ has any arbitrary value; then, according to the foregoing formulæ, we shall have,

$$
\begin{aligned}
& x=\xi+\frac{\mathbf{S}(a e)}{\mathbf{S}\left(a^{2}\right)}, \\
& e=\varepsilon+a \cdot \frac{\mathbf{S}(a e)}{\mathbf{S}\left(a^{2}\right)}, \\
& e^{\prime}=\varepsilon^{\prime}+a^{\prime} \cdot \frac{\mathbf{S}(a e)}{\mathbf{S}\left(a^{2}\right)}, \\
& e^{\prime \prime}=\varepsilon^{\prime \prime}+a^{\prime \prime} \cdot \frac{\mathbf{S}(a e)}{\mathbf{S}\left(a^{2}\right)}, \\
& \text { \&c. }
\end{aligned}
$$

It is evident, from these expressions that $x$ and all the errors $e, e^{\prime}, e^{\prime \prime} \& c$. depend upon one and the same arbitrary quantity $\mathbf{S}(a e)$. If we take the value of $\mathrm{S}(a e)$ in the formula for $e$, and substitute it in the expressions of $e^{\prime}, e^{\prime \prime} \& \mathrm{c}$. we shall get,

$$
\begin{aligned}
& e=\varepsilon+\frac{a}{a}(e-\varepsilon) \\
& e^{\prime}=\varepsilon^{\prime}+\frac{a^{\prime}}{a}(e-\varepsilon) \\
& e^{\prime \prime}=\varepsilon^{\prime \prime}+\frac{a^{\prime \prime}}{a}(e-\varepsilon) \\
& \& c .
\end{aligned}
$$

Which proves that all the errors are deternined when one only is known. The errors therefore are not independent on one another; and this is true of every possible system, excepting only the system $\varepsilon, \varepsilon^{\prime}, \varepsilon^{\prime \prime} \& c$. which is deduced from the rule of the least squares. It is therefore this last system of errors alone that can occur in a set of experiments or observations in which there exists no bias tending regularly one way, and where the error in one case is supposed to have no influence whatever on the error in any other case.

If we make the coefficients $a, a^{\prime}, a^{\prime \prime}$, \&c. all equal to one another, or rather all equal to unit, we shall have the case of a repetition of the same experiment or observation, viz.

$$
\begin{aligned}
& e=x-m \\
& e^{\prime}=x-m^{\prime} \\
& e^{\prime \prime}=x-m^{\prime \prime} \\
& \text { \&c. }
\end{aligned}
$$

where $x$ represents the unknown magnitude to be found, and $m, m^{\prime}, m^{\prime \prime}$, \&c. the experimental values of it. Here the only system of independent errors is determined by the condition $S(\varepsilon)=0$, or, 


$$
\varepsilon+\varepsilon^{\prime}+\varepsilon^{\prime \prime}+\& \text { c. }=0,
$$

which is equivalent to the rule of the arithmetical mean. In every other system the errors have a constant part common to them all, as is evident from what the general expressions become in the particular case under consideration, viz.

$$
\begin{aligned}
& e=\varepsilon+(e-\varepsilon) \\
& e^{\prime}=\varepsilon^{\prime}+(e-\varepsilon) \\
& e^{\prime \prime}=\varepsilon^{\prime \prime}+(e-\varepsilon) \\
& \text { \&c. }
\end{aligned}
$$

Such a set of errors cannot occur unless by the operation of some preponderating cause tending to augment or diminish every error in an equal degree. If there be no bias in the experiments, we must necessarily adopt the only system in which every error is singly determined by its proper quantities independently of all the other errors. The rule of the arithmetical mean is therefore only a particular inference from the general reasoning we have been explaining.

On account of its great practical utility, the method of the least squares has been the subject of much discussion on the continent. In order to prove it recourse has been had to the doctrine of probabilities and the most abstruse researches of the algebraic calculus. But the real grounds of the method are undoubtedly, the general character of the errors, and the properties of the equations of condition. From this last consideration we readily deduce that there is only one system of errors, or perhaps in particular circumstances, only a certain number of systems, that can possibly be consistent with the nature of the experiments. Viewed in this light, the method of the least squares is separated from the laws of chance, and is made to depend on very simple and elementary principles. The application of probability to physical researches rests on other grounds; namely, the general expression of the chance of an error so modified as to lead to rules sufficiently simple for application in practice. Although $I$ have here confined myself to the most simple case of only one correction, yet the principles laid down are general, and are readily applied to several independent corrections by a repetition of similar reasoning.

Sept. 4, 1826.

J. Ivory. 